\title{
Transport measurements on MOVPE-grown InN films
}

\author{
Shang-Chia Chen ${ }^{\mathrm{a}}$, Shih-Kai Lin ${ }^{\mathrm{a}}$, Kun-Ta Wu ${ }^{\mathrm{a}}$, Chao-Ping Huang ${ }^{\mathrm{a}, *}$, Pen-Hsiu Chang ${ }^{\mathrm{b}}$, \\ N.C. Chen ${ }^{\mathrm{b}}$, Chin-An Chang ${ }^{\mathrm{b}}$, Hsian-Chu Peng ${ }^{\mathrm{b}}$, Chuang-Feng Shih ${ }^{\mathrm{b}, \mathrm{c}}$, Kuo-Shung Liu ${ }^{\mathrm{c}}$, \\ Hong-Syuan Wang ${ }^{\mathrm{a}}$, Pu-Tai Yang ${ }^{\mathrm{a}}$, C.-T. Liang ${ }^{\mathrm{a}}$, Y.H. Chang ${ }^{\mathrm{a}}$, Y.F. Chen ${ }^{\mathrm{a}}$ \\ ${ }^{a}$ Department of Physics, National Taiwan University, Taipei 106, Taiwan, ROC \\ ${ }^{\mathrm{b}}$ Institute of Electro-Optical Engineering, Chang Gung University, Kwei-Shan, Tao-Yuan 333, Taiwan, ROC \\ ${ }^{\mathrm{c}}$ Department of Materials Science and Engineering, National Tsing Hua University, Hsinchu 300, Taiwan, ROC
}

Available online 2 April 2005

\begin{abstract}
We have performed electrical transport measurements on $\mathrm{InN}$ films. Our results show that the electron transport in our InN films is metallic-like, that is, within the experimental error the carrier density is temperature-independent over a wide temperature range $(4 \mathrm{~K} \leq T \leq 290 \mathrm{~K})$. At low temperatures, the resistivities of our InN devices appear to saturate and show gradual increase with increasing temperatures. We suggest that residue impurity scattering limits the electron mobility in InN films. We compare our results with existing theoretical models.
\end{abstract}

(C) 2005 Elsevier Ltd. All rights reserved.

Keywords: InN films; Transport measurements

\section{Introduction}

$\mathrm{InN}$, a semiconductor, has been attracting a great deal of both theoretical and experimental interest recently [1]. Advancing growth techniques have made it possible to prepare higher-quality InN samples [2,3], leading to the revision of the fundamental band gap energy from $1.8-2.1 \mathrm{eV}$ to $0.7-0.8 \mathrm{eV}[4,10]$. As a result, the band gap of $\mathrm{InGaN}$ is known to span from the near infrared ( $\mathrm{InN}$ ) to the ultraviolet $(\mathrm{GaN})$ [5]. This allows the entire solar spectrum to be covered by a single material system. Therefore, the alloys of $\mathrm{InN}$ are considered promising materials to be used in a wide range of optoelectronic devices such as light-emitting diodes and solar cells.

In this paper, we report transport measurements on InN films over a wide range of temperature. We show that the electron transport in our InN films is metallic-like [8,9], that is, within the experimental error the carrier density is temperature-independent over a wide temperature range. This is in line with the seminal work by Mahboob et al. [7]

\footnotetext{
* Corresponding author.

E-mail address: ctliang@phys.ntu.edu.tw (C.-P. Huang).
}

0026-2692/\$ - see front matter (c) 2005 Elsevier Ltd. All rights reserved. doi:10.1016/j.mejo.2005.02.038 which showed the Fermi energy is above the conduction band bottom in InN.

\section{Experimental}

The InN samples used in this study were grown with unintentional n-type doping by an Aixtron 200/4RF-S metal-organic vapour phase epitaxy (MOVPE) system [12]. The large lattice mismatch between InN and $\alpha-\mathrm{Al}_{2} \mathrm{O}_{3}(0001)$ substrate $(25 \%)$ can result in an extremely high density of structure defects which lowers the electron mobility in an InN system. One way to overcome this is to insert an intermediate buffer layer [6] between $\alpha-\mathrm{Al}_{2} \mathrm{O}_{3}$ and subsequent InN film. In our case, a GaN buffer layer was used. The thicknesses of all our InN films are approximately $200 \mathrm{~nm}$. The photoluminescence (PL) measurements on our devices showed a broad emission peak around $0.78 \mathrm{eV}$, consistent with those reported in other high-quality $\mathrm{InN}$ systems of comparable electron densities and mobilities. All the electrical transport measurements were performed on cleaved squares $(2-3 \mathrm{~mm})$ on a side. Au or $\mathrm{Al}$ electrodes on the samples were connected to the outer electrodes by conductive silver paste. Four-terminal transport measurements were performed in a continuous-flow $\mathrm{He}^{4}$ system. The low-field electron densities were determined by Hall measurements whereas the resistance and the resistivities of 
our samples were determined by van der Pauw measurements. Data were taken in a rapid succession $(\approx 1 \mathrm{~s})$ while the samples slowly drifted in temperature over the course of several hours. Measurements performed on three devices are presented in this paper. Sample A has a carrier density of $1.23 \times 10^{25} \mathrm{~m}^{-3}$ with a mobility of $1000 \mathrm{~cm}^{2} / \mathrm{Vs}$ at room temperature (RT). At RT, Sample B has a carrier density of $1.37 \times 10^{25} \mathrm{~m}^{-3}$ with a mobility of $980 \mathrm{~cm}^{2} / \mathrm{Vs}$. Sample C has a carrier density of $1.35 \times 10^{25} \mathrm{~m}^{-3}$ with a mobility of $710 \mathrm{~cm}^{2} / \mathrm{Vs}$ at RT.

\section{Results and discussion}

Fig. 1 shows the measured carrier density $n$ as a function of temperature for three different samples A, B and C. We can see that within the experimental errors, the carrier concentrations are temperature-independent, reminiscent of those for a metal. Therefore, our InN systems could be considered degenerate semiconductors in which the Fermi level is above the conduction band bottom over the whole measurement range. This is in line with the data obtained by Mahboob and co-workers. Using the effective mass in InN, we estimate the Fermi energies of Samples A, B and C are 283, 304 and $300 \mathrm{meV}$, respectively. The largest thermal smearing in our system is about $25 \mathrm{meV}$, which is considerably smaller than the Fermi energy in all our samples.

Having demonstrated that $n$ is temperature-independent and therefore our $\mathrm{InN}$ devices are degenerate electron systems [11], we now turn our attention to the resistivity measurements. Fig. 2 shows the measured resistivity as a function of temperature for three different samples A, B and C. We can see that at low temperatures, the measured resistivities tend to saturate. The reason for this is that at low temperatures, electron-phonon scattering is negligible. In this regime, residue impurity and dislocation scattering is temperature-independent for a truly degenerate electron system, causing the resistivity to saturate. With increasing temperature, electron-acoustic phonon scattering increases,

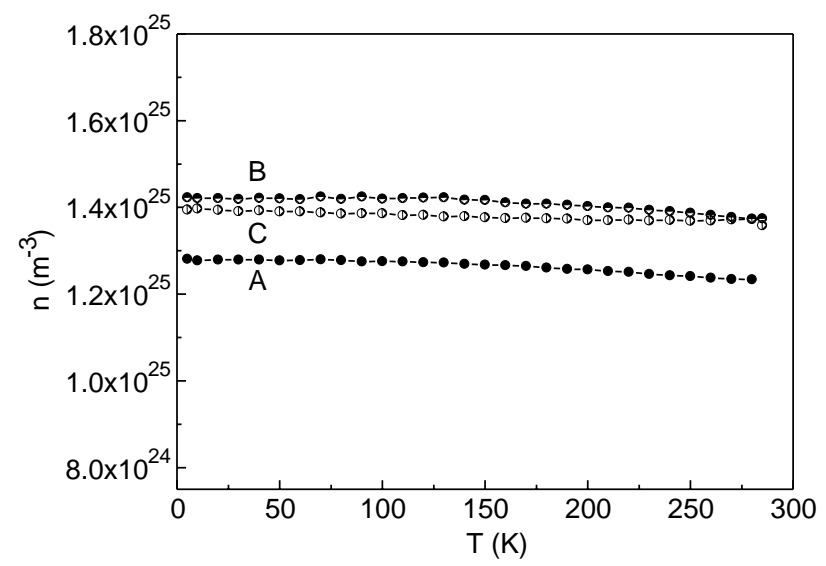

Fig. 1. The measured carrier densities $n$ as a function of temperature for three different samples A, B and C.

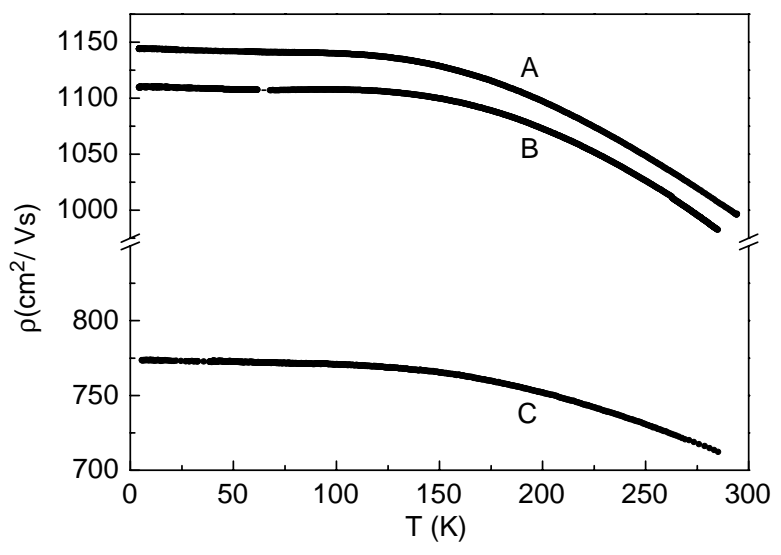

Fig. 2. The measured electrical resistivities $\rho_{x x}$ as a function of temperature for three different samples A, B and C.

decreasing the electron mobility in our system. We can see that the ratio $\rho_{\mathrm{RT}} / \rho_{4 \mathrm{~K}}$ is $\approx 1.1$. This is in sharp contrast to that $\left(>10^{3}\right)$ for a ultra-high-quality degenerate two-dimensional electron gas at the interface of the modulation-doped GaAs/AlGaAs heterostructure [12]. Our results suggest that the dominant scattering mechanism is residue imperfection scattering since $\rho_{\mathrm{RT}}$ is only $\approx 10 \%$ larger than $\rho_{4 \mathrm{~K}}$.

In order to provide further understanding of the transport properties of our InN films, we have measured the mobilities of all our samples. We calculate the mobility using the averaged carrier concentration through the relation $\mu=1 / n \rho e$. This is the quantity which is generally referred to in charge transport in semiconductors. Fig. 3 shows the measured mobilities as a function of temperature for three different samples A, B and C. We can see that the mobility decreases with increasing temperature. This is consistent with the fact that electron-acoustic phonon scattering increases with increasing temperatures.

\section{Conclusions}

In conclusion, we have performed transport measurements on InN films over a wide temperature range.

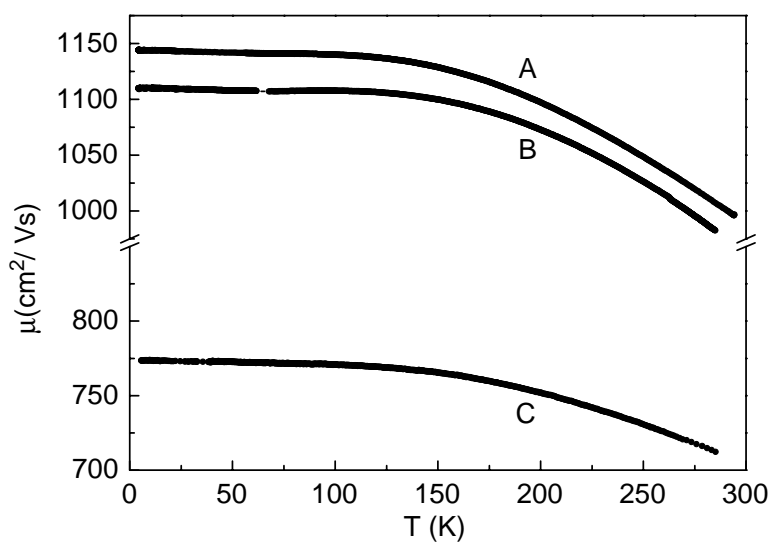

Fig. 3. The measured mobilities $\mu$ as a function of temperature for three different samples A, B and C. 
Our results show that the carrier densities are, within the experimental error, temperature-independent, similar to those in a metal. Therefore, our InN systems can be considered as a degenerate electron system in which the Fermi level is much higher than the conduction band bottom over the whole measurement range. Our results suggest that over wide temperature range, the dominant scattering mechanism is residue imperfection scattering since $\mu_{\mathrm{RT}}$ is only $\approx 10 \%$ smaller than $\mu_{4 \mathrm{~K}}$. Our experimental data clearly demonstrate that in order to obtain higher-mobility InN systems, one needs to substantially reduce the number of background impurities and defects during the growth. Our data demonstrate that electron transport in $\mathrm{InN}$ is metallic-like.

\section{Acknowledgements}

This work was funded by the NSC, Taiwan. We thank C.C. Chang, T.-M. Chen, Y.-H. Chiu, and K.S. Cho for their experimental help.

\section{References}

[1] For a review, see A.G. Bhuiyan, A. Hashimoto, A. Yamamoto, J. Appl. Phys. 94 (2003) 2779 and references therein.

[2] H. Lu, W.J. Schaff, J. Huang, H. Wu, W. Yeo, A. Pharkya, L.F. Eastman, Appl. Phys. Lett. 77 (2000) 2548.

[3] H. Lu, W.J. Schaff, J. Hwang, H. Wu, G. Koley, L.F. Eastman, Appl. Phys. Lett. 79 (2001) 1489.

[4] J. Wu, W. Walukiewicz, K.M. Yu, J.W. Ager III, E.E. Haller, H. Lu, W.J. Schaff, W. Saito, Y. Nanishi, Appl. Phys. Lett. 80 (2002) 3967.

[5] J. Wu, W. Walukiewicz, K.M. Yu, J.W. Ager III, E.E. Haller, H. Lu, W.J. Schaff, Appl. Phys. Lett. 80 (2002) 4741.

[6] H. Lu, W.J. Schaff, L.F. Eastman, C.E. Stutz, Appl. Phys. Lett. 82 (2003) 1736.

[7] I. Mahboob, T.D. Veal, C.F. McConville, H. Lu, W.J. Schaff, Phys. Rev. Lett. 92 (2004) 036804.

[8] M. Konagai, T. Yamada, T. Akatsuka, K. Saito, E. Tokumitsu, K. Takahashi, J. Cryst. Growth 98 (1989) 167.

[9] A. Yamada, Y. Jia, M. Konagi, K. Takahashi, Jpn. J. Appl. Phys. 28 (1989) L2284.

[10] J. Wu, W. Walukiewicz, W. Shan, K.M. Yu, J.W. Ager III, E.E. Haller, H. Lu, W.J. Schaff, Phys. Rev. B 66 (2002) 201403.

[11] D.C. Look, H. Lu, W.J. Schaff, J. Jasinski, Z. Liliental-Weber, Appl. Phys. Lett. 80 (2002) 258.

[12] C.-A. Chang, C.-F. Shi, N.C. Chen, P.-H. Chang, K.-S. Liu, Phys. Status Solidi C 1 (2004) 2559 